\title{
viewpoints
}

\section{VACCINE DEVELOPMENT — AN IDEAL TEMPERED BY REALITY}

\section{Progress with the vaccines we currently have ...}

The progress of immunisation with many diseases is impeded by practical and biological obstacles. For example, 80 or more types of pneumococci and other bacteria cause pneumonia, and presently available vaccine is ineffective in combating multiple etiologic agents with such wide geographic distribution.

Influenza offers somewhat the same problem, with so many viruses involved, some of which remain to be discovered. The disability and morbidity statistics are often overexaggerated, and actual statistical evidence indicates little difference between epidemic and non-epidemic years in the numbers of people disabled by influenza. Seemingly highly epidemic mortality rates, linked to secondary pneumonia infections, are no longer valid as a reason to immunise the older population. In fact, available data support the premise that those who do succumb, would probably have died later in the year from other causes. Even the morbidity rate for young people remains unaffected by annual influenza vaccinations. One must keep in mind that influenza vaccines only contain pre-epidemic viruses, they offer very little current epidemic protection.

There is hope, though, for almost complete elimination of measles and rubella throughout the world, and for the elimination of poliomyelitis in underdeveloped tropical areas. For the most part, the available vaccines are effective. Well planned and executed mass vaccination programs are needed to '. . . break the chain of transmission.'

Clinical trials are desperately needed to test the new inhalation measles vaccines. Such a method of vaccination is far more practical for large numbers of non-medical personnel to administer.

\section{$\ldots$ and the potential of vaccines under development}

Recent identification of rotaviruses may result in developing a vaccine for acute childhood diarrhoea, a disease which annually kills about 5-10 million children in Third World countries. But once again, such a vaccine may be rendered impotent by the multiple variations of bacterial and viral agents which cause diarrhoea. Simple, inexpensive home therapy programmes would be the best method towards reducing its crippling and fatal effects.

Recurring viral infections, such as genital, ocular, and facial herpes seem to affect more people than any other chronic infection. By its very nature of not providing natural immunity from natural infection, little hope exists in finding an effective vaccine.

Attenuated, live virus vaccine research has been a little disappointing so far. However, wider and more sustained immune protection might be achieved from live influenza $A$ virus than from inactivated vaccines, but only if the current pandemic virus were included, and only if a substantial portion of the world's population could be vaccinated quickly. Then there might be some chance of preventing new antigenic forms from developing. Attenuated live virus vaccine research is also underway to combat hepatitis and respiratory syncytial virus, a major cause of bronchiolitis and pneumonia among infants, but the prospects are not promising at present for the development of an effective vaccine against either of these diseases. However, the attenuated varicella-zoster vaccine developed in Japan, and extensively studied over the last 6 years, looks most promising, for virtually the entire human population of this planet sooner or later contracts varicella-zoster virus, with zoster complications occurring in $8 \%$. Furthermore, in chronically sick children, or those receiving cancer chemotherapy, chickenpox can be fatal.

Unfortunately, ‘ . . the effort to confirm the excellent results reported by the Japanese investigators has been too small, and too slow and uncoordinated when one considers how much misery could be prevented by the eradication of this disease of the human heritage by the proper mass use of an effective vaccine.'

Sabin. A.B.: Journal of the American Medical Association 246: 236 (17 Jul 1981) 